Supporting Information

\title{
Elasticity Reinforcement in
}

\section{Propylene-Ethylene Random Copolymer}

\section{Stretched at Elevated Temperature in Large}

\section{Deformation Regime}

Jiayi Zhao ${ }^{1}$, Yingying Sun ${ }^{2}$,Yongfeng Men ${ }^{1, *}$

1. State Key Laboratory of Polymer Physics and Chemistry, Changchun Institute of Applied Chemistry, Chinese Academy of Sciences, University of Chinese Academy of Sciences, Renmin Street 5625, 130022 Changchun, P.R. China

2. ExxonMobil Asia Pacific Research \& Development Co., Ltd, 1099 Zixing Road, Minhang district, 200241 Shanghai, P.R. China 

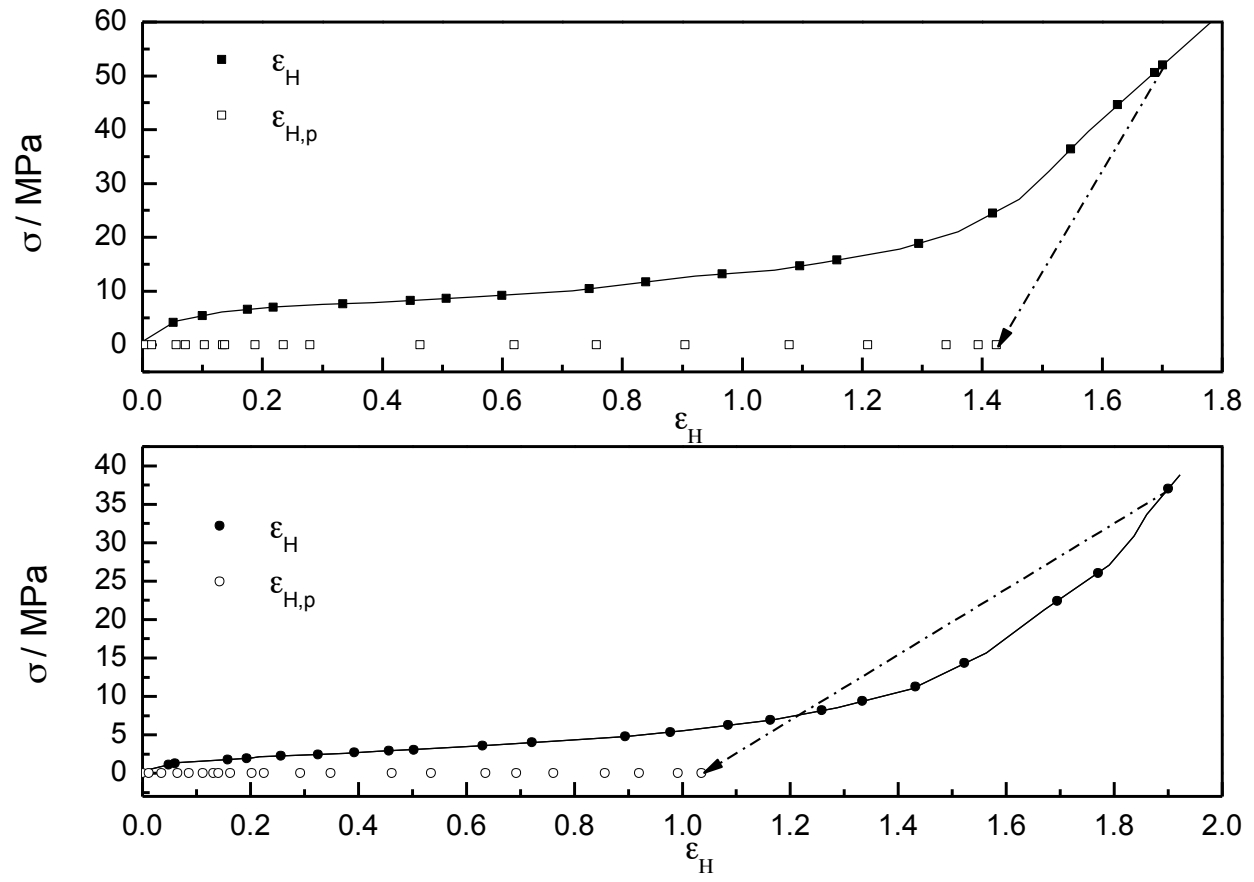

Figure S1. Raw step-cycle results of iPPcoE12 aging for 180 days stretched at room temperature (top) and $63{ }^{\circ} \mathrm{C}$ (bottom). The solid points located on the true stress-strain curves and the hollow ones describe the stretching and relaxing state, respectively.

In step-cycle experiment, the sample was stretched step-by-step at a constant speed. After each step, the crosshead speed was inverted to contract the sample until a stress of zero was achieved. Thereupon, the sample was extended again at this given speed, until it reached another preset larger strain. To overcome heterogeneous deformation distribution over the sample, we recorded photographs of the sample at each step so that the total strain and the remaining plastic strain after complete unloading can be calculated using the width of the sample at certain place of the sample (here the position where X-rays illuminated). Therefore, data of retraction and re-stretching processes were not completely recorded as the emphasis here is the decomposition of 
the total strain into plastic and elastic ones which requires the states of the samples at the initial stretched state and the final relaxed state only. Figure S1 collected all data points we have collected for the "step-cycle" experiments. To show the heterogeneous deformation over the samples, we present in Figure S2 photographs of the samples under stretching and in fully relaxed states.
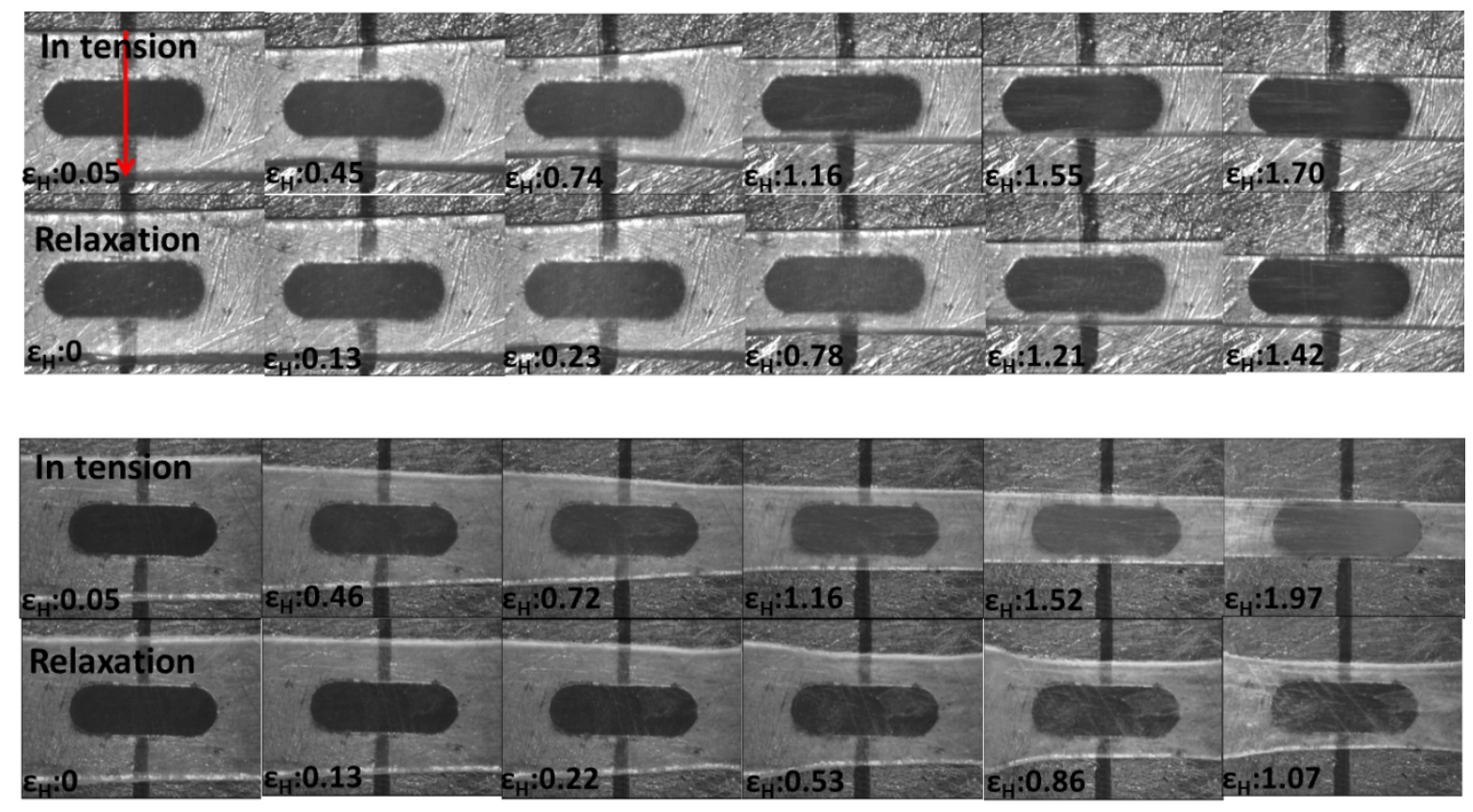

Figure S2. Selected photographs during step-cycle tests of iPPcoE12 aged for 180 days stretched at room temperature (top) and $63{ }^{\circ} \mathrm{C}$ (bottom). 